\title{
O contexto de trabalho de Agentes Comunitários de Saúde: a relação do conteúdo do trabalho com variáveis sociodemográficas
}

\author{
Ariane dos Santos Hoppe ${ }^{1}$ \\ Amanda Côrrea dos Santos ${ }^{2}$ \\ Leni Dias Weigelt ${ }^{3}$ \\ Luciane Maria Schmidt Alves ${ }^{3}$ \\ Suzane Beatriz Frantz Krug ${ }^{4}$
}

\section{RESUMO}

O objetivo do estudo foi investigar a associação do perfil sociodemográfico com a organização, condições e relações socioprofissionais de trabalho de agentes comunitários de saúde (ACS). Estudo exploratório-descritivo, do tipo quantitativo, com 251 ACS de 13 municípios da $13^{\mathrm{a}}$ Coordenadoria Regional de Saúde/RS. Os dados foram coletados através de um questionário sociodemográfico e do "Inventário de Trabalho e Riscos de Adoecimento". Após, foram organizados no software SPSS 20.0 e analisados os valores de média de cada variável. O nível de significância adotado foi de $p \leq 0,05$. Os resultados expressam que o fator "Organização do Trabalho" obteve relação com a idade, o município que o agente comunitário de saúde pertence, tipo de estratégia em que o mesmo está inserido considerando Estratégia de Saúde da Família ou Estratégia de Agentes Comunitários de Saúde - e a área em que atua - urbana ou rural. No fator "Relações Socioprofissionais", houve relação com o município e o tipo de estratégia que o profissional pertence. No fator "Condições de Trabalho", identificou-se relação com a escolaridade do agente comunitário de saúde, o município e área em que atua. Os resultados apontam que há relação estatisticamente significativa entre o perfil sociodemográfico e o contexto de trabalho de ACS.

Palavras-chave: Agentes Comunitários de Saúde. Atenção Básica à Saúde. Condições de Trabalho. Saúde do Trabalhador.

\begin{abstract}
The aim of this study was to investigate the association of the sociodemographic profile with the organization, conditions and socio-professional relations of work of community health agents (CHA). This is an exploratory descriptive study, of the quantitative type, with 251 CHA from 13 municipalities of the 13th Regional Health Coordination/RS. The data were collected through a sociodemographic quiz and from the "Work Inventory and Hazard Risks". Afterwards, the data were organized in the SPSS 20.0 software and were analyzed the average value of each variable. The adopted level of significance was of $p \leq 0,05$. The results express that the factor "Work organization" was related to age, the municipality that the community health agent belongs to, the type of strategy in which they are inserted - considering Family Health Strategy or Community Health Agents Strategy - and the area in which they operate urban or rural. In the "Socioprofessional Relations" factor, there was a relationship with the municipality and the type of strategy that the professional belongs to. In the "Working Conditions" factor, a relationship was identified with the community health agent's schooling,

\footnotetext{
${ }^{1}$ Aluna do Curso de Enfermagem da Universidade de Santa Cruz do Sul - UNISC.

${ }^{2}$ Aluna do Curso de Odontologia da Universidade de Santa Cruz do Sul.

${ }^{3}$ Professoras do Departamento de Enfermagem e Odontologia na Universidade de Santa Cruz do Sul.

${ }^{4}$ Professora no Departamento de Enfermagem e Odontologia e no Mestrado de Promoção em Saúde da

Universidade de Santa Cruz do Sul. <skrug@unisc.br>
} 
the municipality and the area in which they operate. The results indicate that there is a statistically significant relationship between the sociodemographic profile and the work context of CHA.

Keywords: Community Health Agents. Basic Health Care. Work conditions. Worker's health.

\section{INTRODUÇÃO}

O cenário de atuação dos profissionais da Atenção Básica em Saúde (ABS) é permeado por diferentes demandas e desafios no que tange à organização e às condições de trabalho. Nesse sentido, devem ser também considerados os riscos laborais presentes no ambiente de trabalho, sejam eles químicos, ergonômicos, físicos ou biológicos (MAISSIAT et al., 2015).

O contexto de trabalho compreende a forma como o trabalho é organizado, as relações socioprofissionais existentes e as condições do mesmo. Refere-se ao ritmo e distribuição de tarefas, normas, cobranças, integração, comunicação, apoio entre os profissionais, ambiente e materiais disponíveis para o trabalho. Quando não encontra-se adequado às necessidades do indivíduo, pode transformar-se em um potencial fator de sobrecarga física ou psíquica ao profissional (MENDES; ARAÚJO, 2012).

No âmbito da ABS, o Agente Comunitário de Saúde (ACS) é um trabalhador que não possui formação na área da saúde e necessita residir na área de abrangência da Estratégia de Saúde da Família (ESF) em que atua. Estudos descrevem uma realidade laboral composta por inúmeras adversidades nesse trabalho, evidenciando a pouca capacitação do mesmo para a resolutividade das demandas e ações, o que, por muitas vezes, ocasiona desvio de suas competências, implicando em excesso de atribuições e perda da especificidade do seu trabalho (RIBEIRO; AMARAL; STALIANO, 2015; CARNEIRO; MARTINS, 2015; ROSA; BONFANTI; CARVALHO, 2012).

Além disso, observam-se condições de trabalho precárias como a excessiva carga de trabalho, inadequada remuneração, exposição a riscos ambientais sem a disponibilização de Equipamentos de Proteção Individual (EPI) necessários e falta de acesso a capacitações e formações técnicas adequadas. Estes fatores podem afetar a qualidade de vida desse trabalhador, gerando efeito insalubre à sua saúde (CARNEIRO; MARTINS, 2015; SANTOS et al., 2016).

Desse modo, compreender o contexto de trabalho em que esses profissionais estão inseridos e a relação com variáveis pessoais e profissionais dos mesmos, torna-se importante para melhorias nas condições de trabalho. Haja vista que, dessa forma, dar-se-á visibilidade a 
aspectos que necessitam modificações, implicando reflexões e revisões da forma como o trabalho do ACS está organizado, facilitando o desenvolvimento das suas atividades cotidianas (SANTOS et al., 2016; ALCÂNTARA; ASSUNÇÃO, 2016).

A partir disso, objetivou-se investigar a associação do perfil sociodemográfico com a organização, condições e relações socioprofissionais de trabalho de agentes comunitários de saúde dos municípios da $13^{\text {a }}$ Coordenadoria Regional de Saúde do Rio Grande do Sul.

\section{FUNDAMENTAÇÃO TEÓRICA}

Na década de 70, a concepção de promoção à saúde discutida na $1^{\text {a }}$ Conferência Internacional sobre Cuidados Primários em Saúde chega ao Brasil em um período de repressão e censura, na conjuntura da ditadura militar. O processo de manifestações sociais e luta pela democracia no período originaram diversos movimentos populares sendo, no que tange à saúde, o Movimento de Reforma Sanitária (MRS) o mais expressivo. O MRS mobilizou diversos setores da no intuito de concentrar forças na busca por melhorias nas condições e no acesso à saúde, sendo de extrema importância para a criação do Sistema Único de Saúde (SUS) (COTTA et al., 2013; MONTEIRO; PREVITALI, 2011).

Algumas conquistas, a partir desta mobilização social, foram asseguradas pela Constituição Federal de 1988, art. 196, que define a saúde como "direito de todos e dever do Estado, garantido mediante políticas sociais e econômicas que visem à redução do risco de doença e de outros agravos e ao acesso universal e igualitário às ações e serviços para sua promoção, proteção e recuperação" (BRASIL, 1988). A constituição de 1988 transformou a saúde em direito de cidadania e possibilitou a criação do Sistema Único de Saúde (SUS) e, posteriormente, em 1991, a criação do Programa de Agentes Comunitários de Saúde (PACS) (PAIVA; TEIXEIRA, 2014; DURÃO et al., 2009).

O Programa de Agentes Comunitários de Saúde (PACS) surge a partir de experiências bem-sucedidas no Ceará, com o objetivo de melhorar a qualidade de vida e saúde da população. Em 1994 é criado o Programa Saúde da Família (PSF), atualmente denominado de Estratégia de Saúde da Família (ESF) (SANTOS, 2011). A partir da criação destas estratégias, as equipes de saúde, através do trabalho interdisciplinar, passam a ter um olhar mais abrangente do processo saúde-doença. A ESF propõe a substituição do modelo de saúde curativo centrado no médico por um sistema descentralizado com foco na promoção à saúde e à prevenção de doenças, envolvendo o usuário na atenção à saúde. Neste sentido, a atuação dos Agentes Comunitários de Saúde (ACS) nas ESFs configura-se como atividade importante para a consolidação desta nova concepção de saúde, uma vez que interage diretamente com a 
comunidade em sua área de atuação e torna-se um elo entre os saberes científicos e populares (VIDAL; MOTTA; SIQUEIRA-BATISTA, 2015).

Este trabalhador tem a possibilidade de interagir com os indivíduos diretamente nos espaços familiares, durante as visitas domiciliares, e identificar suas peculiaridades a partir da visualização de suas condições de vida. (VIDAL; MOTTA; SIQUEIRA-BATISTA, 2015; BRASIL, 1999).

De acordo com a portaria $\mathrm{n}^{\circ} 2.488$, de 21 de outubro de 2011, os ACS têm como atribuições realizar o cadastro das pessoas de sua microárea e manter os dados atualizados, desenvolver ações que visem à integração dos serviços de saúde com a população adstrita e as unidades de saúde, desempenhar atividades de prevenção de doenças e agravos e promoção da saúde, além de ações educativas e de vigilância à saúde (BRASIL, 2011). As atividades do ACS, apesar de regidas por uma base legal, podem variar de acordo com o território em que esse se insere, uma vez que suas ações são redefinidas pelos gestores de saúde de modo a adequá-las a realidade local (BRASIL, 2003).

Uma particularidade do trabalho do ACS é a obrigatoriedade em residir no mesmo local onde desempenha suas atividades, conforme estabelecido pela Lei $n^{\circ} 10.507$ de 2002 . Isso proporciona a esse trabalhador o contato constante com os usuários, aproximando as ações de saúde do contexto domiciliar (SANTOS et al., 2011; BRASIL, 2002). Entretanto, essa proximidade ao mesmo tempo em que facilita a atividade desse trabalhador, também o expõe a uma dinâmica laboral particular, deixando-o suscetível a várias condições que interferem em seu bem-estar (PAULA et al., 2015; RESENDE et al., 2011).

A mediação realizada pelo ACS entre usuários e equipe de saúde é característica do seu processo de trabalho que pode interferir negativamente na saúde deste trabalhador, em decorrência da deposição de expectativas por parte de ambos universos. A deposição de anseios e expectativas sobre estes trabalhadores por parte da comunidade e da equipe, as dificuldades de relacionamento entre equipe de saúde e população, os riscos ambientais a que estão expostos durante as visitas domiciliares e a falta de boas condições de trabalho são fatores citados em diversos estudos com ACS como dificultadores ou causadores de angústia e sofrimento (BRAND; ANTUNES; FONTANA, 2010; GALAVOTE et al., 2011; ROSA; BONFANTI; CARVALHO, 2012; SANTOS; VARGAS; REIS, 2014; JUSTO; GOMES; SILVEIRA, 2015). Nesse sentido, compreende-se que tanto o ambiente de trabalho quanto a forma como se organiza o processo de trabalho do ACS configuram-se como potentes estressores (JUSTO; GOMES; SILVEIRA, 2015). 


\section{MATERIAIS E MÉTODOS}

O presente estudo caracteriza-se como exploratório-descritivo do tipo quantitativo, um recorte da pesquisa "Sofrimento no trabalho de Agentes Comunitários de Saúde: um estudo nos municípios da $13^{\text {a }}$ Coordenadoria Regional de Saúde - RS", desenvolvida pelo Grupo de Estudos e Pesquisa em Saúde (GEPS) da Universidade de Santa Cruz do Sul, no período de 2014-2015, contemplada no edital FAPERGS/MS/CNPq/SESRS n.002/2013 e Programa Pesquisa para o SUS - PPSUS - 2013/2015.

Os sujeitos pesquisados foram 251 ACS de 34 unidades de ESF e cinco Estratégias de Agentes Comunitários de Saúde (EACS) dos 13 municípios do âmbito da $13^{\text {a }}$ Coordenadoria Regional de Saúde, $28^{\mathrm{a}}$ Região de Saúde do Rio Grande do Sul. Essa região de saúde se constitui de 13 municípios localizados na região dos Vales e abrange uma população de 343.858 habitantes (IBGE, 2014). Os municípios são, em sua maioria, formados por extensas áreas rurais, com atividades voltadas para a agricultura e pecuária. As regiões urbanas destacam-se pela atividade das indústrias e do comércio, o que confere à região expressiva importância econômica. No que se refere à atenção básica, nos 13 municípios totaliza-se 46 Unidades Básicas de Saúde (UBS) e 44 com ESF.

A coleta de dados foi realizada com base em dois instrumentos: um questionário sociodemográfico com 9 questões e o formulário "Inventário de Trabalho e Risco de Adoecimento" (ITRA), validado em estudos de Mendes e Ferreira (2007). O ITRA é um inventário composto por quatro dimensões (escalas) que busca investigar os fatores presentes na atividade laboral que podem contribuir para o processo de adoecimento no trabalho e seus efeitos sobre o trabalhador, por meio da avaliação da inter-relação entre trabalho e adoecimento.

Para este estudo, utilizou-se a primeira dimensão do ITRA denominada Escala de Avaliação do Contexto de Trabalho (EACT) que avalia o conteúdo do trabalho, envolvendo organização, relações socioprofissionais e condições de trabalho. Trata-se de uma escala do tipo Likert de 5 pontos composta por 31 questões e dividida em três fatores: organização do trabalho, condições de trabalho e relações socioprofissionais.

Os dados foram organizados através do software SPSS 20.0 e analisados os valores de média de cada variável. Para a verificação de associação significativa $(\mathrm{p} \leq 0,05)$ entre as variáveis utilizou-se o teste não-paramétrico de U de Mann-Whitney. A pesquisa foi aprovada pelo Comitê de Ética em Pesquisa da Universidade de Santa Cruz do Sul - UNISC sob o protocolo 713.475 . 


\section{RESULTADOS E DISCUSSÃO}

O perfil dos sujeitos desse estudo expressa uma realidade de ACS majoritariamente do sexo feminino $(92,8 \%, \mathrm{n}=233)$, com idade entre 31 a 40 anos $(37,5 \%, \mathrm{n}=94)$ e com estado civil casado $(48,6 \%, \mathrm{n}=122)$. Sobre a escolaridade, $54,2 \%(\mathrm{n}=136)$ têm ensino médio completo e $13,2 \%(n=33)$ estavam cursando o ensino superior. Em relação ao tipo de estratégia, grande parte dos ACS era vinculada a ESF $(59,8 \%, \mathrm{n}=150)$ e o restante a EACS $(40,2 \%, \mathrm{n}=101)$, com predomínio de atuação em área urbana $(50,2 \%, \mathrm{n}=126)$, seguido de área rural $(43,8 \%, \mathrm{n}=110)$ e em ambas as áreas $(6 \%, \mathrm{n}=15)$.

Na Tabela 1 são apresentados os cruzamentos estatísticos realizados entre o fator “Organização do Trabalho” da EACT e as variáveis do perfil sociodemográfico dos ACS.

Tabela 1 - Cruzamento das variáveis sociodemográficas com o fator Organização do Trabalho

\begin{tabular}{lc}
\hline \multicolumn{1}{c}{ Cruzamentos } & Organização do Trabalho \\
\hline Idade & 0,001 \\
Sexo & 0,738 \\
Estado civil & 0,190 \\
Escolaridade & 0,504 \\
Filhos & 0,546 \\
Dependentes & 0,643 \\
Tipo de estratégia & 0,001 \\
Área de atuação & 0,000 \\
Município & 0,000 \\
\hline
\end{tabular}

Fonte: Dados da pesquisa, 2016.

Os resultados demonstram que o fator "Organização do Trabalho" obteve relação significativa com as variáveis "idade", "município" que o ACS pertence, "tipo de estratégia" em que o mesmo está inserido - considerando ESF ou EACS - e a "área de atuação" - urbana ou rural.

As variáveis sociodemográficas cruzadas com o fator "Relações Socioprofissionais" estão apresentadas na tabela 2. 
Tabela 2 - Cruzamento das variáveis sociodemográficas com o fator Relações Socioprofissionais

\begin{tabular}{lc}
\hline \multicolumn{1}{c}{ Cruzamentos } & Relações Socioprofissionais \\
\hline Idade & 0,083 \\
Sexo & 0,865 \\
Estado civil & 0,318 \\
Escolaridade & 0,518 \\
Filhos & 0,317 \\
Dependentes & 0,587 \\
Tipo de estratégia & 0,015 \\
Área de atuação & 0,265 \\
Município & 0,000 \\
\hline
\end{tabular}

Fonte: Dados da pesquisa, 2016.

Evidenciou-se que no fator "Relações Socioprofissionais" da escala houve relação significativa com as variáveis "município" que o ACS pertence e "tipo de estratégia" que integra.

Em relação ao cruzamento das variáveis sociodemográficas com o fator "Condições de trabalho", os resultados podem ser observados na tabela 3 .

Tabela 3 - Cruzamento das variáveis sociodemográficas com o fator Condições de Trabalho

\begin{tabular}{lc}
\hline \multicolumn{1}{c}{ Cruzamentos } & Condições de Trabalho \\
\hline Idade & 0,348 \\
Sexo & 0,900 \\
Estado civil & 0,680 \\
Escolaridade & 0,013 \\
Filhos & 0,063 \\
Dependentes & 0,811 \\
Tipo de estratégia & 0,635 \\
Área de atuação & 0,028 \\
Município & 0,011 \\
\hline
\end{tabular}

Fonte: Dados da pesquisa, 2016. 
Identificou-se no terceiro fator, "Condições de trabalho", relação significativa com as variáveis "escolaridade" do ACS, "município" que pertence e "área de atuação".

A associação significativa da variável "idade" com o fator "organização do trabalho" pode relacionar-se às expectativas criadas pelo jovem trabalhador acerca de sua atuação profissional. Isso, tendo em vista que os mais jovens, ao adentrarem no mundo do trabalho, apresentam pouca experiência para o enfrentamento de situações conflitantes do dia-a-dia laboral, ocasionando um embate entre a realidade e o que era esperado (MAISSIAT et al., 2015). Além disso, para os trabalhadores com mais idade, existe uma maior propensão para a manifestação de dores musculoesqueléticas, visto que as longas caminhadas e a forma como o trabalho de ACS está organizado contribuem para o surgimento dessas limitações físicas (SANTOS et al., 2016).

Além da idade, a escolaridade também tem importante influência na posição do indivíduo na sociedade, haja vista que as mesmas podem implicar em mais ou menos potencialidades para o enfrentamento de aspectos do ambiente externo (ALCÂNTARA; ASSUNÇÃO, 2016), o que pode explicar a relação estatística encontrada nesse estudo entre a variável "escolaridade" e o fator "condições de trabalho".

No estudo de Rosa, Bonfanti e Carvalho (2012) é discutida a relação da parcialidade da qualificação e da precária formação continuada dos ACS com a origem do sofrimento psíquico, dado que, a partir disso, percebe-se a existência de relações de poder inerentes às condições de trabalho apresentadas no local pesquisado. Nesse sentido, no estudo de Mota e David (2010) evidenciou-se que houve um significativo aumento em todas as faixas de escolaridades entre os ACS pesquisados. Com isso, pode-se inferir que o ACS como membro da comunidade e que, por vezes, não possui uma bagagem cultural ou técnica pertinentes à área da saúde, ao defrontar-se com novas experiências de trabalho e de informações, busca diferentes alternativas de profissionalização. Além disso, Santos et al. (2016) destacam que indivíduos com maior nível de escolaridade compreendem de forma mais adequada os conceitos de saúde, o cotidiano e a si mesmo, contribuindo, dessa forma, para o melhor desempenho do trabalho.

Encontrou-se, nesse estudo, relação significativa da variável "tipo de estratégia" que o ACS integra com os fatores "organização do trabalho" e "relações socioprofissionais" da EACT. Esse achado indica que existem diferenças na forma como os trabalhadores de ESF e EACS percebem as relações com outros profissionais e comunidade e na forma como está organizado o trabalho em cada equipe. 
Cabe salientar que o trabalho em equipe representa uma característica importante das equipes de ESF, tendo em vista que as mesmas são compostas por diferentes profissionais, o que contribui para a realização de uma assistência integral e multidisciplinar às famílias da comunidade. Dessa forma, observa-se que as equipes de ESF proporcionam ao ACS um sentimento de segurança pelo apoio encontrado em outros profissionais para a resolução das demandas identificadas nas visitas domiciliares e pela possibilidade de discussão dos problemas cotidianos, gerando novos aprendizados (SANTANA et al., 2009; PERES et al., 2011). Possivelmente, esse sentimento de apoio apresenta-se de forma desigual para os ACS de EACS, uma vez que, na região estudada, os mesmos não integram uma equipe multidisciplinar, sendo coordenados por um profissional enfermeiro.

Outra associação significativa encontrada nesse estudo refere-se à variável "área de atuação" do ACS, considerando área urbana ou rural, com os fatores "organização do trabalho" e "condições de trabalho". A partir de outros estudos (BAPTISTINI; FIGUEIREDO, 2014; GOMES et al., 2015), evidencia-se a influência das características do local de atuação no desenvolvimento do trabalho de ACS, dado que, nesses estudos, foram encontradas realidades urbanas com altos índices de violência e com exposição a doenças infecciosas e áreas rurais com exposição a ataques de animais e dificuldades no acesso às residências. Nesse sentido, essas péssimas condições de trabalho representam importantes riscos à saúde do ACS, podendo implicar em agravos físicos e psicológicos aos mesmos. A diferença do trabalho em área urbana e área rural remete a necessidade de planejamentos de acordo com cada realidade, principalmente no que tange às características sociais e geográficas do local (BAPTISTINI; FIGUEIREDO, 2014; GOMES et al., 2015).

No presente estudo, identificou-se uma associação significativa da variável "município" com os três fatores da EACT: organização do trabalho, relações socioprofissionais e condições de trabalho. A partir disso, evidencia-se que o contexto de trabalho de ACS apresenta-se de forma distinta nos diferentes municípios. Esse fato também foi observado em outros estudos como os de Rosa, Bonfanti e Carvalho (2012) e de Gomes et al. (2015) que apontam realidades nas quais os ACS são utilizados nas campanhas eleitorais, realizam funções administrativas, de recepção e de enfermagem. Os mesmos, muitas vezes, são cobrados na realização de atividades para as quais não foram capacitados e com prazos insuficientes para sua execução, distorcendo as reais atribuições desses trabalhadores (GOMES et al., 2015).

A forma da gestão do trabalho de ACS apresenta grande influência no surgimento de agravos de ordem psicológica nesses profissionais, principalmente no que se refere à 
sobrecarga de trabalho, à alta demanda psicológica na realização das tarefas e à ausência de treinamento, como é visto nos estudos de Alcântara e Assunção (2016) e de Gomes et al. (2015). Ademais, é expressa através de falas a falta de suporte institucional do poder público e da gestão local, além da falta de reconhecimento profissional (OLIVEIRA et al., 2010).

Ainda, observam-se realidades com inadequação e/ou insuficiência de materiais no cotidiano laboral de ACS, tanto de equipamentos para proteção individual, como de instrumentos para suprir as necessidades de trabalho, contribuindo para a manifestação de estresse no trabalho (GOMES et al., 2015; OLIVEIRA et al., 2010). Portanto, destaca-se que a disponibilização de equipamentos de trabalho necessários, conforme a realidade e necessidade dos serviços de saúde, torna a atividade do ACS menos desgastante e favorece a realização de um atendimento de qualidade para os usuários (VOGT et al., 2012).

\section{CONCLUSÃO}

Através desse estudo, evidenciou-se a existência de associação significativa do perfil sociodemográfico com a organização do trabalho, relações socioprofissionais e condições de trabalho de ACS nos municípios de abrangência da $13^{\text {a }}$ Coordenadoria Regional de Saúde do Rio Grande do Sul. Enfatiza-se que a variável "município" foi a única que obteve associação significativa com os três fatores da Escala de Avaliação do Contexto de Trabalho, o que nos leva a refletir a existência de possíveis dificuldades vivenciadas por esses trabalhadores, o apoio oferecido pela gestão e as condições de trabalho.

Dessa forma, aponta-se a importância de uma gestão focada nas necessidades do trabalhador, haja vista que as variáveis que apresentaram associação significativa com os fatores da escala traduzem realidades distintas e devem ser pensadas individualmente. Além disso, cabe destacar que a forma como o contexto de trabalho apresenta-se para o indivíduo pode influenciar significativamente no desempenho do trabalho e na saúde do trabalhador.

\section{REFERENCIAS}

ALCÂNTARA, M. A.; ASSUNÇÃO, A. A. Influência da organização do trabalho sobre a prevalência de transtornos mentais comuns dos agentes comunitários de saúde de Belo Horizonte. Revista Brasileira de Saúde Ocupacional, São Paulo, v. 41, e. 2, 2016. Acesso em: 26 jan. 2017. DOI: http://dx.doi.org/10.1590/2317-6369000106014.

BAPTISTINI, R. A.; FIGUEIREDO, T. A. M. Agente comunitário de saúde: desafios do trabalho na zona rural. Ambiente e Sociedade, São Paulo, v. 17, n. 2, p. 53-70, 2014. Acesso em: 10 out. 2016. DOI: http://dx.doi.org/10.1590/S1414-753X2014000200005 
BRAND, C. I.; ANTUNES, R. M.; FONTANA, R. T. Satisfações e insatisfações no trabalho do agente comunitário de saúde. Cogitare Enfermagem, Curitiba, v. 15, n. 1, p. 40-47, 2010. Acesso em: 26 jan. 2017. DOI: http://dx.doi.org/10.5380/ce.v15i1.17143

BRASIL. Constituição da República Federativa do Brasil, de 5 de outubro de 1988. Diário Oficial [da] República Federativa do Brasil, Brasília, DF, 6 out. 1988. Seção I. Disponível em: <http://www.planalto.gov.br/ccivil_03/constituicao/constituicao.htm〉. Acesso em: 24 jan. 2017.

BRASIL. Decreto $\mathrm{n}^{\circ}$ 3.189, de 4 de outubro de 1999. Fixa diretrizes para o exercício da atividade de Agente Comunitário de Saúde (ACS), e dá outras providências. Diário Oficial [da] República Federativa do Brasil, Brasília, DF, 5 out. 1999. Seção 1, p. 1. Disponível em: <http://presrepublica.jusbrasil.com.br/legislacao/111303/decreto-3189-99>. Acesso em: 30 jan. 2017.

BRASIL. Lei n. 10.507, de 10 de julho de 2002. Cria a profissão de agente comunitário de saúde, e dá outras providências. Diário Oficial da República Federativa do Brasil, Brasília, 11 jul. 2002. Seção 1. Disponível em: <http://www.saude.mt.gov.br/atencao-asaude/arquivo/1398/normatizacoes> Acesso em: 30 jan. 2017.

BRASIL. Ministério da Saúde. Portaria n. ${ }^{\circ} 2.488$, de 21 de outubro de 2011. Aprova a Política Nacional de Atenção Básica, estabelecendo a revisão de diretrizes e normas para a organização da Atenção Básica, para a Estratégia de Saúde da Família (ESF) e o Programa de Agentes Comunitários de Saúde (PACS). Diário Oficial da União, 2011. Disponível em: $<$ http://bvsms.saude.gov.br/bvs/saudelegis/gm/2011/prt2488_21_10_2011.html>. Acesso em: 30 jan. 2017.

BRASIL. Ministério da Saúde. Programa Saúde da Família: ampliando a cobertura para consolidar a mudança do modelo de atenção básica. Revista Brasileira de Saúde Materno Infantil, Recife, v. 3, n. 1, p. 113-125, 2003. Acesso em: 4 fev. 2017. DOI: http://dx.doi.org/10.1590/S1519-38292003000100013.

CARNEIRO, C. C. G.; MARTINS, M. I. C. Novos modelos de gestão do trabalho no setor público de saúde e o trabalho do agente comunitário de saúde. Trabalho, Educação e Saúde, Rio de Janeiro, v. 13 n. 1, p. 45-65, 2015. Acesso em: 15 fev. 2017. DOI: http://dx.doi.org/10.1590/1981-7746-sip00029

COTTA, R. M. M. et al. Políticas de saúde: desenhos, modelos e paradigmas. Viçosa: UFV, 2013.

DURÃO, A. V. et al. Analisando o processo de qualificação do Agente Comunitário de Saúde (ACS). In: MONKEN, M.; DANTAS, A. V. Estudos de politécnica e saúde. Rio de Janeiro: EPSJV, v. 4, 2009. p. 191-219. Disponível em: <http://pesquisa.bvs.br/brasil/resource/pt/eps2596>. Acesso em: 28 jan. 2017.

GALAVOTE, H. S. et al. Desvendando os processos de trabalho do agente comunitário de saúde nos cenários revelados na Estratégia Saúde da Família no município de Vitória (ES, Brasil). Ciência \& Saúde Coletiva, Rio de Janeiro, v. 16, n. 1, p. 231-40, 2011. Acesso em: 15 fev. 2017. DOI: http://dx.doi.org/10.1590/S1413-81232011000100026 
GOMES, M. F. et al. Riscos e agravos ocupacionais: percepções dos agentes comunitários de saúde. Revista de pesquisa: cuidado é fundamental (Online), Rio de Janeiro, v. 7, n. 4, p. 3574-3586, 2015. Disponível em: <http://saudepublica.bvs.br/pesquisa/resource/pt/bde27212>. Acesso em: 15 out. 2016.

IBGE. Instituto Brasileiro de Geografia e Estatística. Estimativa da população residente nos municípios brasileiros. 2014. Disponível em: <http://www.ibge.gov.br/home/estatistica/populacao/estimativa2014/estimativa_dou.shtm>. Acesso em: 16 fev. 2017.

JUSTO, C. M. P.; GOMES, M. H. A.; SILVEIRA, C. Limites e imposições dos instrumentos de controle do trabalho de agentes comunitários de saúde da Estratégia Saúde da Família. Saúde e Sociedade, São Paulo, v. 24, n. 2, p. 594-606, 2015. Acesso em: 16 fev. 2017. DOI: http://dx.doi.org/10.1590/S0104-12902015000200016

MAISSIAT, G. S. et al. Contexto de trabalho, prazer e sofrimento na atenção básica em saúde. Revista Gaúcha de Enfermagem, Porto Alegre, v. 36, n. 2, p. 42-49, 2015. Acesso em: 10 out. 2016. DOI: http://dx.doi.org/10.1590/1983- 1447.2015.02.51128

MENDES, A. M. B.; ARAÚJO, L. K. R. Clínica psicodinâmica do trabalho: o sujeito em ação. Curitiba: Juruá, 2012.

MENDES, A. M. B.; FERREIRA, M. C. Inventário sobre Trabalho e Riscos de Adoecimento - ITRA: instrumento auxiliar de diagnóstico de indicadores críticos no trabalho. Capítulo 5. In: MENDES, A. M. B. (Org.) Psicodinâmica do trabalho: teoria, método e pesquisa. São Paulo: Casa do Psicólogo, p. 111-126, 2007.

MONTEIRO, M. A. G. S.; PREVITALI, F. S. A política de formação profissional dos agentes comunitários de saúde: limites e possibilidades de construção de sujeitos críticos. Revista Labor, Fortaleza, v. 1, n. 5, p. 141-163, 2011. Disponível em: <http://www.periodicos.ufc.br/index.php/Labor/article/view/6641>. Acesso em: 27 jan. 2017

MOTA, R. R. A.; DAVID, H. M. S. L. A crescente escolarização do agente comunitário de saúde: uma indução do processo de trabalho?. Trabalho, Educação e Saúde, Rio de Janeiro, v. 8, n. 2, p. 229-248, 2010. Acesso em: 10 out. 2016. DOI: http://dx.doi.org/10.1590/S198177462010000200004

OLIVEIRA, A. R. et al. Satisfação e limitação no cotidiano de trabalho do agente comunitário de saúde. Revista Eletrônica de Enfermagem, Goiânia, v. 12, n. 1, p. 28-36, 2010. Acesso em: 20 fev. 2017. DOI: http://dx.doi.org/10.5216/ree.v12i1.9511.

PAIVA, C. H. A.; TEIXEIRA, L. A. Reforma sanitária e a criação do Sistema Único de Saúde: notas sobre contextos e autores. História, Ciências, Saúde - Manguinhos, Rio de Janeiro, v. 21, n. 1, p. 15-35, 2014. Acesso em: 27 jan. 2017. DOI: http://dx.doi.org/10.1590/S010459702014000100002

PAULA, I. R. et al. Capacidade para o trabalho, sintomas osteomusculares e qualidade de vida entre agentes comunitários de saúde em Uberaba, Minas Gerais. Saúde e Sociedade, São Paulo, v. 24, n.1, p.152-164, 2015. Acesso em: 28 jan. 2017. DOI: http://dx.doi.org/10.1590/S0104-12902015000100012 
PERES, C. R. F. B. et al. O Agente Comunitário de Saúde frente ao processo de trabalho em equipe: facilidades e dificuldades. Revista da Escola de Enfermagem da USP, São Paulo, v. 45, n. 4, p. 905-911, 2011. Acesso em: 15 fev. 2017. DOI: http://dx.doi.org/10.1590/S008062342011000400016

RESENDE, M. C. et al. Saúde mental e ansiedade em agentes comunitários que atuam em saúde da família em Uberlândia (MG, Brasil). Ciência \& Saúde Coletiva, Rio de Janeiro, v. 16, n. 4, p. 2115- 2122, 2011. Acesso em: 28 jan. 2017. DOI: http://dx.doi.org/10.1590/S141381232011000400011 .

RIBEIRO, S. F. R.; AMARAL, P. J. V.; STALIANO, P. Sofrimento Psíquico do Agente Comunitário de Saúde: revisão bibliográfica. Revista Laborativa, São Paulo, v. 4, n. 1, p. 7996, 2015. Disponível: <http://ojs.unesp.br/index.php/rlaborativa/article/view/1172〉. Acesso em: 20 jan. 2017.

ROSA, A. J.; BONFANTI, A. L.; CARVALHO, C. S. O sofrimento psíquico de Agentes Comunitários de Saúde e suas relações com o trabalho. Saúde e Sociedade. São Paulo, v. 21, n. 1, p. 141-152, 2012. Acesso em: 10 out. 2016. DOI: http://dx.doi.org/10.1590/S010412902012000100014

SANTANA, J. C. B. et al. Agente Comunitário de Saúde: percepções na Estratégia Saúde da Família. Cogitare Enfermagem, Curitiba, v. 14, n. 4, p. 645-652, 2009. Acesso em: 15 fev. 2017. DOI: http://dx.doi.org/10.5380/ce.v14i4.16377

SANTOS, F. A. A. S. et al. Fatores que influenciam na qualidade de vida dos agentes comunitários de saúde. Acta Paulista de Enfermagem, São Paulo, v. 29, n. 2, p. 191-197, 2016. Acesso em: 10 fev. 2017. DOI: http://dx.doi.org/10.1590/1982-0194201600027

SANTOS, I. E. R. Estresse ocupacional em agentes comunitários de saúde da cidade de Aracaju-Sergipe. 2011. 62 f. Dissertação (Programa de Pós-Graduação em Saúde e Ambiente - Mestrado) - Universidade Tiradentes, Aracaju, 2011. Disponível em: <http://ppg.unit.br/wpcontent/uploads/sites/6/2016/05/Dissertacao-MSA-Italo-docx.pdf >. Acesso em: 27 jan. 2017.

SANTOS, I. E. R.; VARGAS, M. M; REIS, F. P. Estressores laborais em agentes comunitários de saúde. Revista Psicologia, Organizações e Trabalho, Brasília, v. 14, n. 3, p. 324-35. 2014. Disponível em: <http://pepsic.bvsalud.org/scielo.php?script=sci_arttext\&pid=S198466572014000300008>. Acesso em: 28 jan. 2017.

SANTOS, K. T. et al. Agentes comunitários de saúde: perfil adequado à realidade do programa de saúde da família?. Ciência \& Saúde Coletiva, Rio de Janeiro, v. 16, supl. 1, p. 1023-1028, 2011. Acesso em: 29 jan. 2017. DOI: http://dx.doi.org/10.1590/S141381232011000700035

VIDAL, S. V.; MOTTA, L. C. S.; SIQUEIRA-BATISTA, R. Agentes comunitários de saúde: aspectos bioéticos e legais do trabalho vivo. Saúde e Sociedade, São Paulo, v. 24, n. 1, p. 129 140, 2015. Acesso em: 26 jan. 2017. DOI: 10.1590/S0104-12902015000100010 
VOGT, M. S. et al. Cargas físicas e psíquicas no trabalho do Agente Comunitário de Saúde. Cogitare Enfermagem, Curitiba, v. 17, n. 2, p. 297-303, 2012. Acesso em: 11 out. 2016. DOI: http://dx.doi.org/10.5380/ce.v17i2.23559

Como citar esse documento: HOPPE, Ariane dos Santos et al. O contexto de trabalho de Agentes Comunitários de Saúde: a relação do conteúdo do trabalho com variáveis sociodemográficas. Revista Jovens Pesquisadores, Santa Cruz do Sul, v. 7, n. 1, jan. 2017. ISSN 2237-048X. Disponível em: <https://online.unisc.br/seer/index.php/jovenspesquisadores/article/view/9301>. Acesso em: ... doi: http://dx.doi.org/10.17058/rjp.v7i1.9301. 